OPEN

SUBJECT AREAS:

SENSORS AND

BIOSENSORS

DESIGN, SYNTHESIS AND PROCESSING

Received

19 November 2013

Accepted

2 January 2014

Published

22 January 2014

Correspondence and requests for materials should be addressed to

J.H. (jiaxing-huang@ northwestern.edu)

* These authors contributed equally to this work.

\section{Pencil Drawn Strain Gauges and Chemiresistors on Paper}

\author{
Cheng-Wei Lin*, Zhibo Zhao*, Jaemyung Kim \& Jiaxing Huang
}

Department of Materials Science and Engineering, Northwestern University 2220 Campus Drive, Evanston, IL, 60208, USA.

Pencil traces drawn on print papers are shown to function as strain gauges and chemiresistors. Regular graphite/clay pencils can leave traces composed of percolated networks of fine graphite powders, which exhibit reversible resistance changes upon compressive or tensile deflections. Flexible toy pencils can leave traces that are essentially thin films of graphite/polymer composites, which show reversible changes in resistance upon exposure to volatile organic compounds due to absorption/desorption induced swelling/ recovery of the polymer binders. Pencil-on-paper devices are low-cost, extremely simple and rapid to fabricate. They are light, flexible, portable, disposable, and do not generate potentially negative environmental impact during processing and device fabrication. One can envision many other types of pencil drawn paper electronic devices that can take on a great variety of form factors. Hand drawn devices could be useful in resource-limited or emergency situations. They could also lead to new applications integrating art and electronics.

$\mathrm{n}$ recent years, paper-based electronics have garnered significant attention due to the potential to produce flexible, thin, low-cost, portable, and environmentally-friendly products including antennae ${ }^{1}$, touch pads ${ }^{2}$, microfluidic devices ${ }^{3}$, displays ${ }^{4}$, sound sources ${ }^{5}$, printed circuit boards ${ }^{6}$, and sensors ${ }^{7,8}$. Unlike traditional substrates such as silicon, glass, and/or plastic, paper fibers offer a naturally porous environment which provides an increased deposition area and allows the formation of composites using materials deposited within the cellulose fibers?. Thus, devices that can benefit from large contact surfaces such as energy storage devices ${ }^{10-14}$, solar cells ${ }^{15}$, and ultraviolet (UV) sensors ${ }^{16}$ have been developed to utilize these advantages.

In most of these paper-based devices, writing inks made of expensive materials such as carbon nanotubes and metal nanoparticles are typically used through extensive processing techniques. These drawing materials are often dispersed in liquids and deposit traces on papers after solvent evaporation. On the other hand, a pencil, an everyday commodity, can easily make graphite depositions on various substrates by dry drawing (Figure 1a). The main component of the pencil lead is fine graphite powders bound together by clays. Figure $1 \mathrm{c}$ is a photo showing cross-sectional view of pencil lead. When observed under scanning electron microscope (SEM, Figure 1d), graphite platelets can be clearly seen. The platelets appear wavy due to the compression they experienced during manufacturing. When pencil traces are drawn on paper, friction between the pencil lead and the paper rubs off graphite particles which in turn adhere to the paper fibers. Thus pencils can be viewed as an easily deployable form of ultrafine graphite particles. Also, pencil traces can be regarded as conductive thin films made of percolated graphite particle network on paper (SEM, Figure 1b), which can take on arbitrary shapes and patterns. Pencil traces are quite stable against moisture, chemicals and UV irradiation. Although carbon-based materials have been extensively explored for the aforementioned printed device applications $s^{17-20}$, interests in pencil-base approaches has just begun to emerge ${ }^{21-24}$. Pencil traces have been mainly used as passive conductive elements in many devices, such as electrode contacts for batteries ${ }^{22}$, UV sensors ${ }^{25}$, microfluidic devices ${ }^{26}$, resistors ${ }^{24}$ and transistors ${ }^{23}$. In one recent study, Ren et al. demonstrated that pencil traces can be incorporated as the active components in piezoresistive sensors ${ }^{21}$. They showed that instead of using carbon ink formulated in a laboratory, a commercially available pencil can be used to draw patterns on paper, which then can be connected to an external measurement system through metallic electrodes to construct piezoresistive sensors. Here, we demonstrate that pencil traces can function not only as strain gauges to detect compressive and tensile deflections, but also as chemiresistors sensitive to volatile chemical vapors. Moreover, complete devices can be fabricated without the need for metallic electrodes using only pencil traces drawn from a single type of pencil or a combination of different types of pencils in an all-pencil fabrication process. The pencil-on-paper approach offers a unique method to developing sensing platforms where devices can be fabricated in minutes using nothing more than common office supplies. 

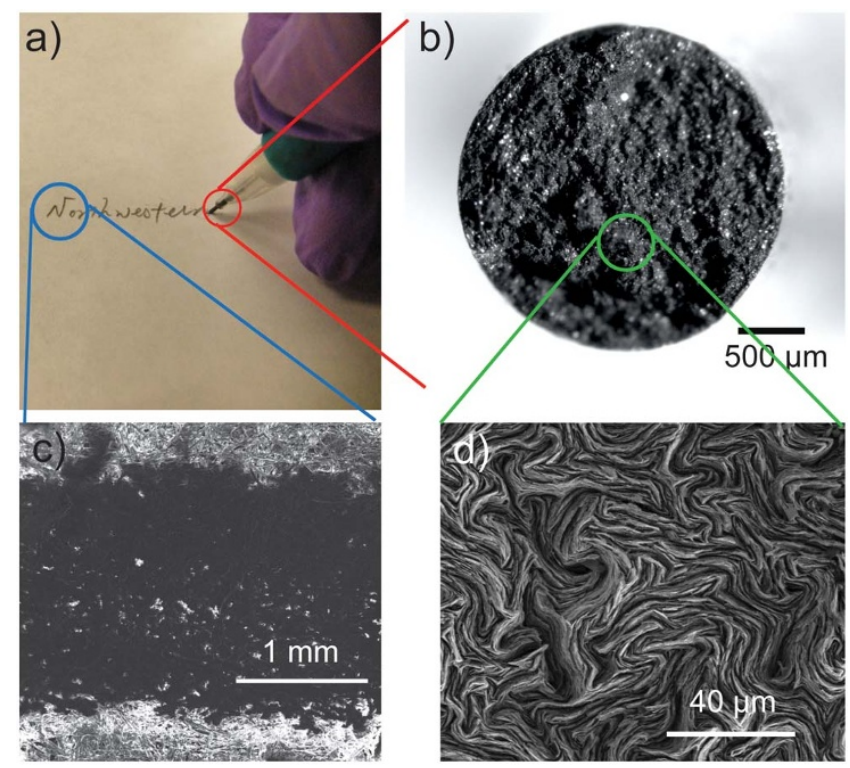

Figure $1 \mid$ (a) Optical image of a propelling pencil loaded with HB leads, writing on paper substrates. (b) Stereomicrograph of a pencil lead. (c) SEM image of a $2 \mathrm{~B}$ pencil trace on paper showing that a continuous carbon particle film was deposited on paper. (d) SEM image of a propelling pencil lead.

\section{Results and Discussion}

Standard pencil leads are composed of fine graphite particles held together by clay binders. Based on the hardness of these leads, pencils are classified on a scale from $9 \mathrm{~B}$ to $9 \mathrm{H}$. The difference in color arises from the different relative fractions of graphite between harder and softer pencil leads. The energy dispersive X-ray spectra (EDS) in Figure $2 \mathrm{a}$ were acquired on the cross-section of six different pencil leads. The intensities of the spectra were normalized based on the carbon peak to highlight the relative fractions of carbon to the clay binder, which is mainly composed of $\mathrm{O}, \mathrm{Mg}, \mathrm{Al}$ and $\mathrm{Si}$. The normalized spectra confirm that harder pencil leads contain a higher proportion of clay binders while softer leads contain a higher proportion of graphite particles. The higher carbon content in the softer leads results in darker traces on paper. Figure $2 b-g$ show optical images of pencil traces drawn on a piece of printing paper, corresponding to six different hardness classes. Under similar writing conditions, the hardest $9 \mathrm{H}$ lead produces the lightest-colored trace while the softest 9B lead produces the darkest one. The electrical conductivity of a pencil trace depends on the quality of the contact between graphite particles in the percolating network. Expansion and contraction of this network, induced by either mechanical stress or chemical interactions, should greatly affect the quality of inter-particle contacts and thus the overall electrical conductivity. Below we show that conductivity of pencil traces indeed respond to external mechanical and chemical stimuli, making them suitable as strain gauges and chemiresistors.

Pencil trace based strain gauges. To test the pencil trace strain gauge, we drew a U-shaped cantilever pattern connected to two solid rectangles on a piece of office paper using a studio pencil (Blick $\left.{ }^{\circledR}\right)$. As shown in Figure $3 \mathrm{a}$, the $U$ part is used as the active sensing beam while the two rectangles are used as contact electrodes. The cantilever is cut out by using scissors. As shown in Figure $3 \mathrm{~b}$, inward and outward deflections of the beam should have different effects on the resistance of the pencil trace. Inward

a)

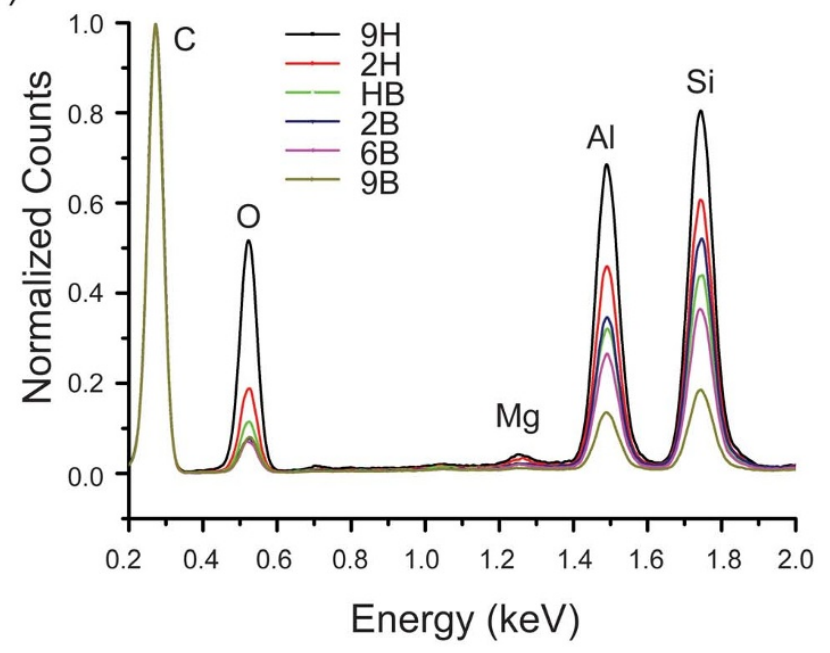

b)

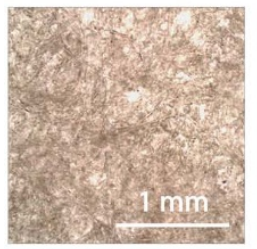

$9 \mathrm{H}$ c)

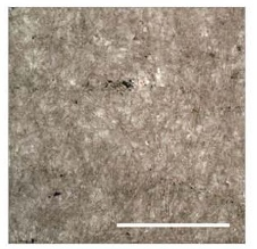

$2 \mathrm{H}$ d)

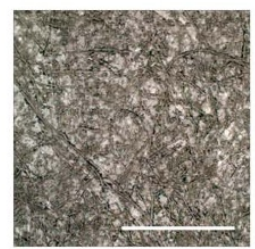

$\mathrm{HB}$ e)

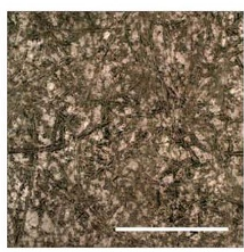

$2 B$ f)

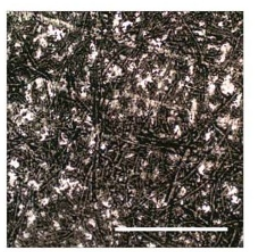

$6 B$ g)

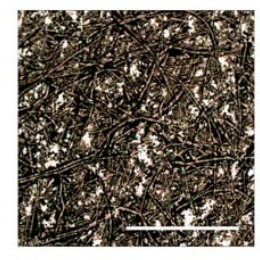

$9 \mathrm{~B}$

Hardest

Medium

Softest

Figure $2 \mid$ (a) EDS spectra of pencil leads correlating hardness with the fraction of carbon. Intensities of all of the spectra were normalized based on the carbon peak. (b-g) Optical microscopy images of traces drawn on the paper substrates from pencils of decreasing hardness. 


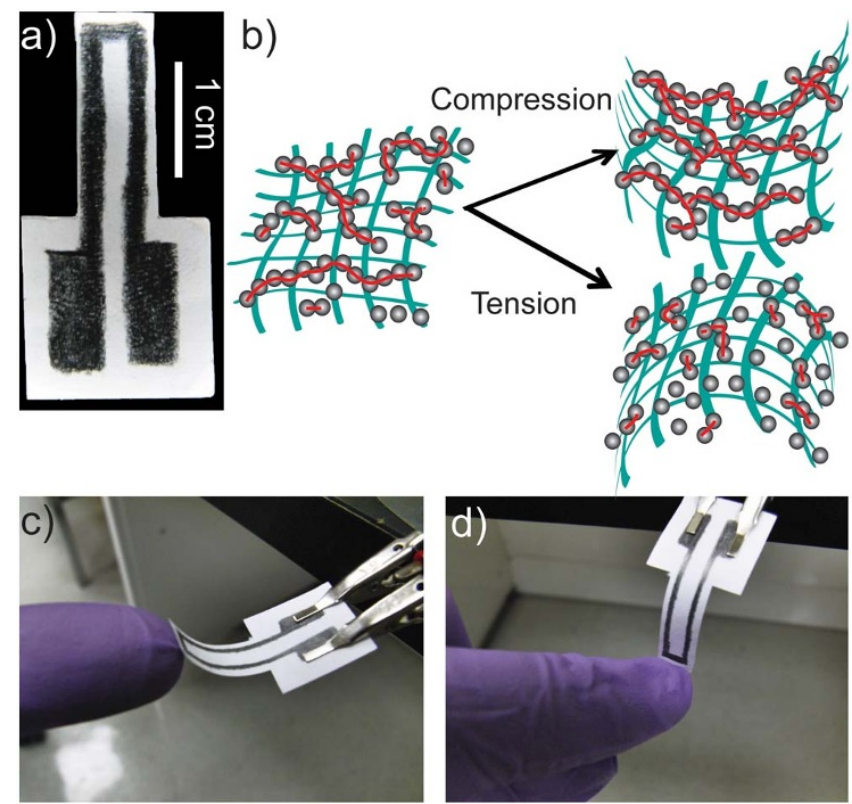

Figure 3 ( (a) A photograph showing the U-shaped pencil trace drawn on a paper beam functioning as a strain gauge. (b) Schematic drawing shows that the number of connected graphite particle chains varies depending on the types of deformation. (c-d) Photograph of the gauge deformed by a single finger in compression and tension, respectively.

a)

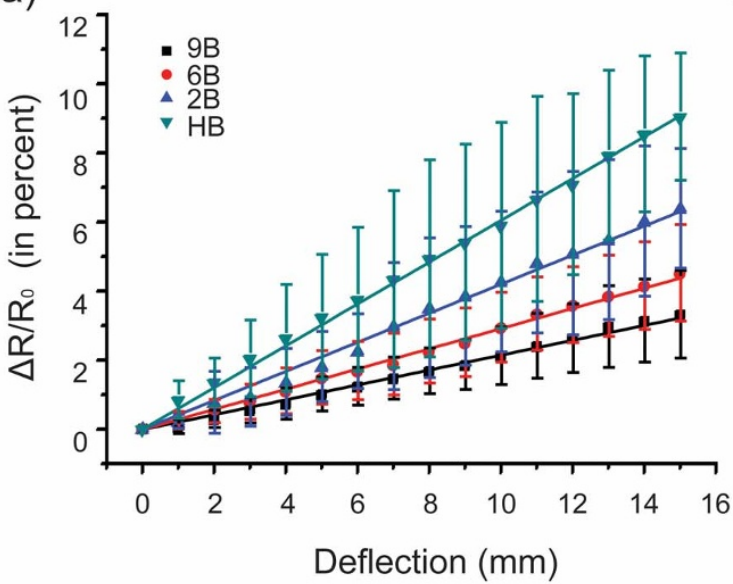

c)

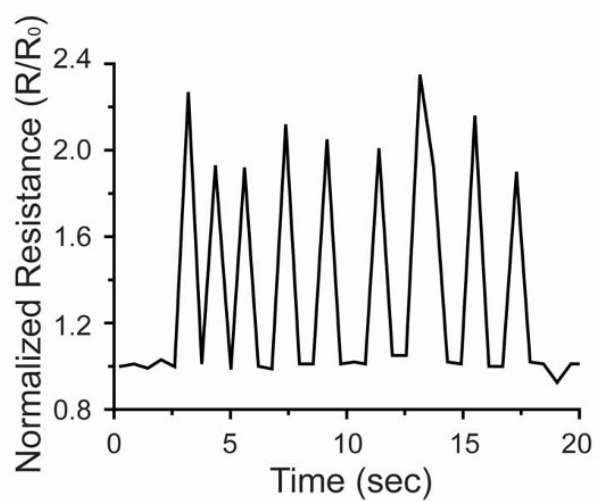

deflection (Figure 3c) effectively compresses the graphite particle network in the two parallel segments of the U-shaped trace, and should lower the resistance. Outward deflection (Figure 3d) should increase the resistance as it stretches the trace. Therefore, the two types of deflections are named compressive and tensile deflections, respectively. Bending tests were done in the geometry similar to Figure $3 \mathrm{c}-\mathrm{d}$ by vertically pressing or lifting the tip of the cantilever. The degree of deflection was quantified by measuring the vertical displacement of the tip. The rectangular contact patterns were fixed on a glass slide so that the deflection only bends the beam. Changes of electrical resistance along the $U$ shaped trace were monitored using a Keithley 2400 source meter connected to the electrodes by toothless alligator clips.

The base resistances of the unstrained devices drawn by $9 \mathrm{~B}, 6 \mathrm{~B}, 2 \mathrm{~B}$, and $\mathrm{HB}$ pencils were measured to be $200 \mathrm{k} \Omega, 500 \mathrm{k} \Omega, 2 \mathrm{M} \Omega$, and $20 \mathrm{M} \Omega$, respectively. As expected, the softer pencil leads with higher graphite content exhibited lower resistances. The base resistances of traces drawn with $2 \mathrm{H}$ and harder pencils were too high to be measured. These four devices were then tested under repeated deflections. Absolute resistances of different devices, $\mathrm{R}$, were normalized by the base resistance of each device, $R_{0}$, and the percent change in resistance, $\Delta R / R_{0}$, was plotted for each device. Under tensile deflection (i.e., outward bending), graphite particles were pulled further apart, disconnecting the conduction pathways, which resulted in higher resistances (Figure 4a). The solid lines represent linear fits to the tensile resistance change-deflection data with equations: $y=$ $0.099 x\left(\mathrm{R}^{2}=0.991\right)$ for the $9 \mathrm{~B}$ device; $y=0.28 x\left(\mathrm{R}^{2}=0.991\right)$ for the $6 \mathrm{~B}$ device; $y=0.48 x\left(\mathrm{R}^{2}=0.991\right)$ for the 2B device, and $y=0.70 x\left(\mathrm{R}^{2}\right.$ $=0.992$ ) for the HB device. Conversely, under compressive deflection, the graphite particles in the trace were pushed closer together, b)

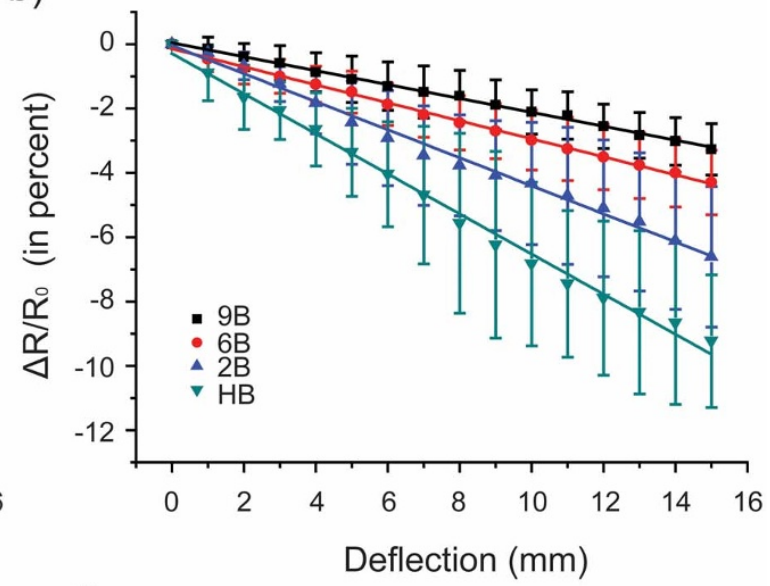

d)

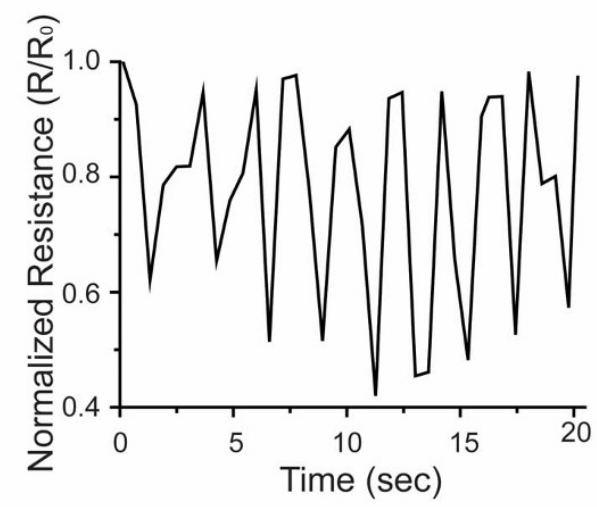

Figure $4 \mid(\mathrm{a}-\mathrm{b})$ Change in normalized resistance vs. deflections for devices drawn with four different types of pencils during compressive and tensile mode of deflections, respectively. (c-d) Repetitive responses under repeated tensile and compressive cycles, respectively. 
facilitating conduction through the percolating network. This indeed decreased the measured resistances (Figure 4b). The solid lines represent linear fits to the compressive resistance change-deflection data with equations: $y=-0.22 x\left(\mathrm{R}^{2}=0.989\right)$ for the 9B device; $y=$ $-0.34 x\left(\mathrm{R}^{2}=0.997\right)$ for the 6B device; $y=-0.38 x\left(\mathrm{R}^{2}=0.991\right)$ for the 2B device; and $y=-0.55 x\left(\mathrm{R}^{2}=0.986\right)$ for the HB device. Within the measured range of deflections, the mean resistance changes were linearly correlated to the mean magnitudes of deflection within around $1 \%$ deviations.

For both compressive and tensile deformation, traces drawn with harder pencils exhibited greater responses, as shown by the steeper slopes of the resistance change-deflection curves in Figure $4 \mathrm{a}$ and $4 \mathrm{~b}$. This indicates that the volume fractions of conductive carbon particles in traces drawn by the harder pencil leads are closer to the percolation threshold than those of softer pencil traces, thus leading to more pronounced "break junction" type of sensing behaviors ${ }^{27}$. Since traces from $\mathrm{HB}$ pencil demonstrated the largest response while $2 \mathrm{H}$ pencil traces were nonconductive, the carbon content of the $\mathrm{HB}$ pencil traces may coincidentally lie just above the percolation threshold.

To test the reusability of the pencil-based touch sensor, we applied short repetitive deflections at regular intervals. Figure $4 \mathrm{c}$ and $4 \mathrm{~d}$ show the sensor responses to these repetitive strains in both tensile and compressive modes. The device consistently exhibited a spike in resistance when a tensile strain was applied followed by a rapid return to the base resistance when the strain was removed. Similarly, the device exhibited a sharp drop in resistance when a compressive strain was applied followed by a rapid return to the base resistance when the strain was removed. Fully reversible responses were observed after tens of bending cycles.

Traces from flexible pencil as chemiresistors. While regular pencils use inorganic clay materials as binder, some specialty pencils use organic and polymeric binders, such as grease pencils for drawing on hard and smooth surfaces or flexible toy pencils that can bend like rubber without breaking the lead (Figure 5a). Extensive works have been done in chemiresistors based on carbon black/polymer composites, in which the percolated carbon particle network is embedded in a volatile organic compound (VOC) sensitive polymer matrix ${ }^{28,29}$. Upon exposure to VOCs, the polymer matrix is swollen, resulting in more separated carbon particles in the percolating network and thus increased resistance (Figure 5e). Since the lead of the flexible pencil is essentially a graphite/ polymer composite, it should be useful as a facile processing tool that can readily generate thin films of graphite/polymer composites (i.e. pencil traces) that can act as chemiresistors for VOCs. The flexible pencil we used consists of graphite particles embedded in a polyvinyl chloride (PVC)-based matrix. The soft polymer binder makes the cross-sectional surface of the flexible pencil lead smoother (Figure $5 \mathrm{~b}$ ) than that of regular pencil leads (Figure 1d). Figure $5 \mathrm{~d}$ shows a chemiresistor drawn on paper, consisting of two layers of pencil traces (Figure $5 \mathrm{c}$ ). The first rectangular shaped layer $(10 \mathrm{~mm} \times 16 \mathrm{~mm})$ was drawn from a flexible pencil as the active sensing element, on top of which a set of interdigitated electrodes (finger width around $0.2 \mathrm{~mm}$ and finger separation $2 \mathrm{~mm}$ ) was drawn using a more conductive regular $\mathrm{HB}$ pencil to better connect the sensing layer to external contacts such as a pair of alligator clips. The use of interdigitated electrodes was found to decrease the initial resistance of the device, and improve the signalto-noise ratio of the measured resistance over time.

To test the response of the device upon exposure to various VOCs, we exposed the device to the vapors above six different solvents using a $250 \mathrm{~mL}$ flask as a head space chamber. The vapor pressures of the six solvents at room temperature $\left(25^{\circ} \mathrm{C}\right)$ are as follows: Acetone 229 torr; methanol 127 torr; ethyl acetate 95 torr; tetrahydrofuran (THF) 162 torr; toluene 28 torr; and hexane 151 torr. Resistance
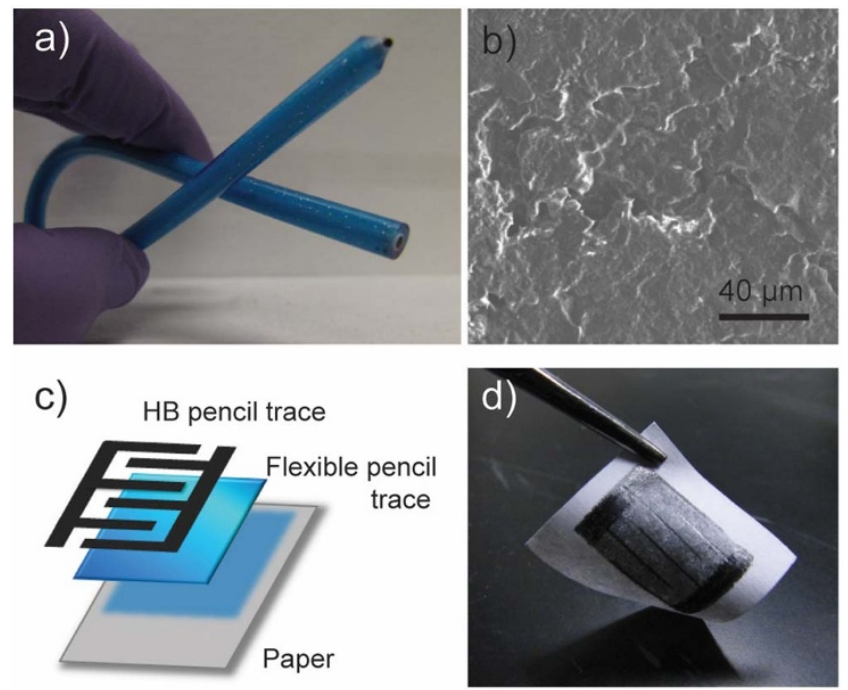

e)

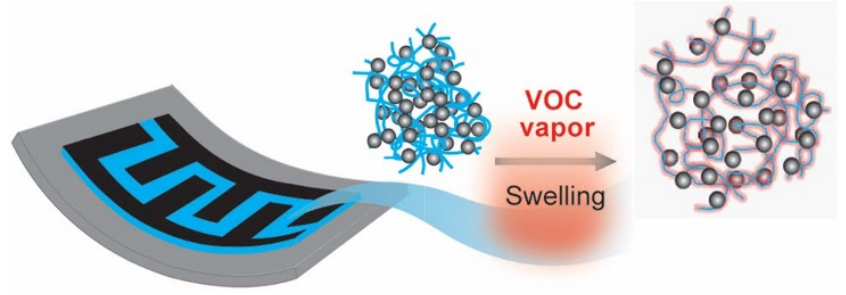

Figure $5 \mid$ (a) Photograph showing a flexible toy pencil. (b) SEM image of the flexible pencil lead. (c) Schematic diagram showing a bilayer chemiresistor. The first rectangular-shaped layer was drawn with a flexible pencil, acting as the active sensing layer, on top of which a set of interdigitated electrode were drawn with an HB pencil to improve electrical contact during measurements. (d) Photograph of the pencildrawn chemiresistor, showing the flexibility of the device. (e) Schematic diagram showing how VOC vapor swells the polymer matrix in the active sensing layer of the device and push the graphite particles apart, where the black dots represent graphite particles.

measurements over time were recorded using a Labview program interfaced with a Keithley 2400 source meter. Figure 6a shows normalized resistance changes $\left(\mathrm{R} / \mathrm{R}_{0}\right)$ of the chemiresistors upon exposure to the above mentioned 6 VOCs above the solvents. The $R / R_{0}$ values for acetone, methanol, ethyl acetate, THF, toluene, and hexane are $1.83 \pm 0.07,1.62 \pm 0.20,1.85 \pm 0.16,3.91 \pm 0.69,1.58 \pm 0.02$, $1.23 \pm 0.03$, respectively. Based on the $R / R_{0}$ value per torr, the sensitivity of the flexible pencil trace to these VOCs decreases from toluene, THF, ethyl acetate, methanol, hexane to acetone, with the relative strength being $7.0,3.0,2.44,1.66,1.02$ to 1.0 , respectively. Since toluene is a processing solvent for PVC and acetone is a nonsolvent ${ }^{30}$, this trend is likely following the solubility of PVC in the organic solvents. Therefore, the differential responses can be attributed to different degrees of swelling of the polymer binders caused by the vapors. This is the foundation of fabricating electronic nose type of sensor arrays ${ }^{28}$. In control experiments, it was verified that traces drawn by regular graphite/clay pencils showed little to no response upon exposure to chemical vapors. Therefore, the responses were indeed attributed to the polymer binder in the flexible pencil traces. Figure $6 \mathrm{~b}$ shows the response of a device upon exposure to acetone vapor over 10 cycles. The device was first equilibrated with the vapor for about $10 \mathrm{~min}$ and removed to the air before testing. This initial equilibration step leads to smoother baselines and higher signal-tonoise ratio. We found that the device consistently exhibited a rapid increase in resistance upon exposure to the acetone vapor followed by a rapid return to its base resistance when the device was 
a)

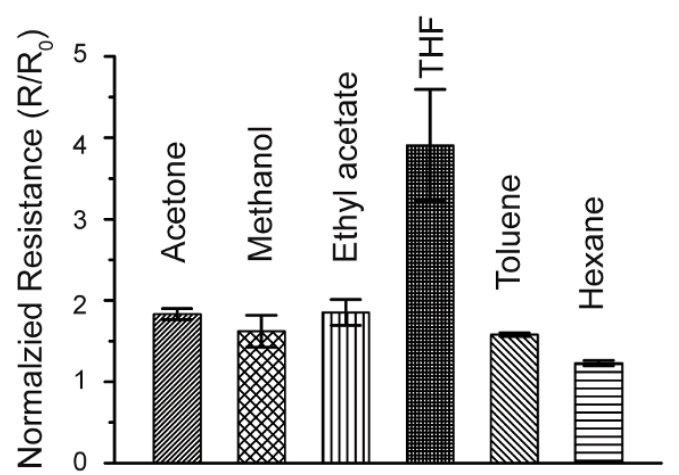

b)

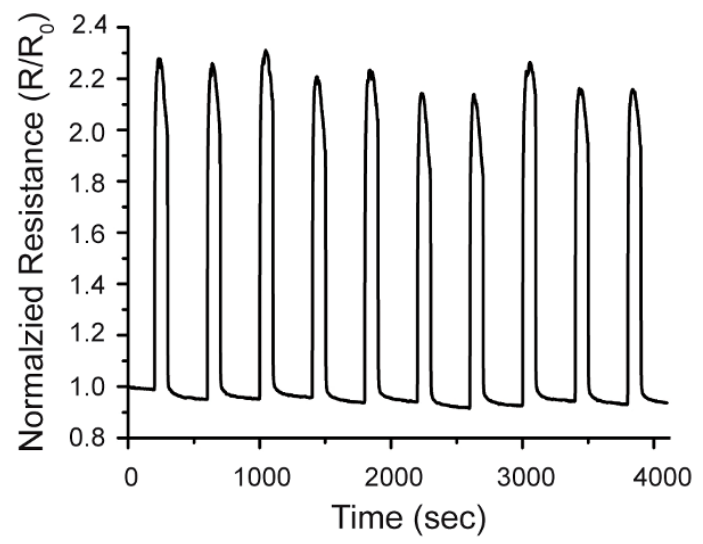

Figure 6 | (a) Normalized resistance changes of the flexible pencil trace devices upon exposure to six different VOCs above the corresponding solvents. (b) Real-time normalized resistance of the device upon cycles (100 sec exposures to acetone vapor), showing good reversibility.

re-exposed to the air. The reversible changes in resistance indicate that the flexible pencil trace can reversibly absorb and desorb acetone vapors. Tests with the other five organic vapors showed similarly reversible responses.

\section{Conclusion and Outlook}

In summary, we have demonstrated two types of functional sensing devices, namely strain gauges and chemiresistors made entirely by pencil traces drawn on paper. These devices are easily manufactured through simple mechanical drawing using commodity pencils on paper substrates. Traces from regular pencils can be readily used as stain sensors based on deformation induced changes in resistance through the percolating graphite particle network. The device performances could be improved by optimizing the geometries of the pencil trace patterns, the darkness of the traces and selecting paper substrates with optimized mechanical properties. The pencil drawn strain gauge can differentiate compressive and tensile signals, suggesting potential touch panel applications, in which the strain sensitive resistors could act as pressure-triggered, digital switching devices for signal input. Traces from flexible toy pencils, essentially thin films of graphite/polymer composites, can act as chemiresistors showing reversible responses upon exposure to VOCs. The work present here highlights pencils as easily deployable forms of graphite fine powders embedded in inorganic or organic matrices. Pencil-onpaper devices are very cheap and extremely simple to make. They are disposable and do not generate potentially negative environmental impact during material processing or device fabrication. They are light, flexible, portable and reusable. By nature, pencil traces are quite durable and do not degrade easily. They can be drawn into arbitrary patterns and take on a great variety of form factors. Pencil drawn devices could be useful in resource-limited or emergency situations, and lead to new applications integrating art and electronics such as when combined with origami.

\section{Methods}

Pencil-drawn strain gauges. To test the pencil-drawn strain gauge, we drew cantilever-shaped sensors comprised of two rectangular electrodes connected by a Ushaped sensing beam as shown in Figure 3a. The strain sensors were fabricated by cutting office paper (EarthChoice ${ }^{\circledR}, 75 \mathrm{~g} \mathrm{~m}^{-2}$ ) using scissors. Both the electrodes and the sensing segments were hand-drawn using commercially available studio drawing pencils (Blick ${ }^{\circledR}$ ). Static bending tests for Figure $4 \mathrm{a}$ and $4 \mathrm{~b}$ were performed by controllable deflection of the U-shaped sensing element. A small glass slide $(18 \mathrm{~mm}$ $\times 18 \mathrm{~mm}$, thickness $\sim 0.15 \mathrm{~mm}$ ) was taped to the back of the cantilever support to ensure the deflection is confined to the beam. Repetitive strains were applied by manually tapping the cantilever beam to a deflection of $\sim 25 \mathrm{~mm}$ as shown in Figure $3 \mathrm{c}$ and $3 \mathrm{~d}$. Resistance changes were monitored using a Keithley 2400 source meter connected to the pencil trace by cables with toothless alligator clips.

Flexible pencil-drawn chemiresistors. The chemiresistor consists of two layers of pencil traces drawn on the paper substrate. The first rectangular layer $(10 \mathrm{~mm} \times$ $16 \mathrm{~mm}$ ) drawn using a flexible pencil acts as the active sensing element, on top of which a set of interdigitated electrodes (finger width about $0.2 \mathrm{~mm}$ and finger separation $2 \mathrm{~mm}$ ) was drawn using an $\mathrm{HB}$ pencil. The use of $\mathrm{HB}$-drawn interdigitated electrodes decreases the initial resistance of the device and increases electrode contact area, making device resistance easier to measure. The device was exposed to different VOC vapors above the corresponding solvents, and the device responses were recorded using a Keithley 4200 Parameter Analyzer.

1. Russo, A. et al. Pen-on-Paper Flexible Electronics. Adv. Mater. 23, 3426-3430 (2011).

2. Mazzeo, A. D. et al. Paper-Based, Capacitive Touch Pads. Adv. Mater. 24, 2850-2856 (2012).

3. Martinez, A. W., Phillips, S. T., Whitesides, G. M. \& Carrilho, E. Diagnostics for the Developing World: Microfluidic Paper-Based Analytical Devices. Anal. Chem. 82, 3-10 (2009).

4. Andersson, P. et al. Active Matrix Displays Based on All-Organic Electrochemical Smart Pixels Printed on Paper. Adv. Mater. 14, 1460-1464 (2002).

5. Tian, H. et al. Graphene-on-Paper Sound Source Devices. ACS Nano 5, 4878-4885 (2011).

6. Siegel, A. C. et al. Foldable Printed Circuit Boards on Paper Substrates. Adv. Funct. Mater. 20, 28-35 (2010).

7. Liu, X., Mwangi, M., Li, X., O’Brien, M. \& Whitesides, G. M. Paper-based piezoresistive MEMS sensors. Lab Chip 11, 2189-2196 (2011).

8. Mirica, K. A., Weis, J. G., Schnorr, J. M., Esser, B. \& Swager, T. M. Mechanical Drawing of Gas Sensors on Paper. Angew. Chem. Int. Ed. 51, 10740-10745 (2012)

9. Johnston, J. H., Moraes, J. \& Borrmann, T. Conducting Polymers on Paper Fibres. Synth. Met. 153, 65-68 (2005).

10. Gwon, H. et al. Flexible energy storage devices based on graphene paper. Energy Environ. Sci. 4, 1277-1283 (2011).

11. Pushparaj, V. L. et al. Flexible energy storage devices based on nanocomposite paper. Proc. Natl. Acad. Sci. 104, 13574-13577 (2007).

12. Nyholm, L., Nyström, G., Mihranyan, A. \& Strømme, M. Toward Flexible Polymer and Paper-Based Energy Storage Devices. Adv. Mater. 23, 3751-3769 (2011).

13. Hu, L., Wu, H., La Mantia, F., Yang, Y. \& Cui, Y. Thin, Flexible Secondary Li-Ion Paper Batteries. ACS Nano 4, 5843-5848 (2010).

14. Weng, Z. et al. Graphene-Cellulose Paper Flexible Supercapacitors. Adv. Energy Mater. 1, 917-922 (2011).

15. Barr, M. C. et al. Direct Monolithic Integration of Organic Photovoltaic Circuits on Unmodified Paper. Adv. Mater. 23, 3500-3505 (2011).

16. Gimenez, A. J., Yáñez-Limón, J. M. \& Seminario, J. M. ZnO-Paper Based Photoconductive UV Sensor. J. Phys. Chem. C 115, 282-287 (2010).

17. Geim, A. K. Graphene: Status and Prospects. Science 324, 1530-1534 (2009).

18. Baughman, R. H., Zakhidov, A. A. \& de Heer, W. A. Carbon Nanotubes-the Route Toward Applications. Science 297, 787-792 (2002).

19. Sreeprasad, T. S. et al. Electron-Tunneling Modulation in Percolating Network of Graphene Quantum Dots: Fabrication, Phenomenological Understanding, and Humidity/Pressure Sensing Applications. Nano Lett. 13, 1757-1763 (2013).

20. Mirica, K. A., Azzarelli, J. M., Weis, J. G., Schnorr, J. M. \& Swager, T. M. Rapid prototyping of carbon-based chemiresistive gas sensors on paper. Proc. Natl. Acad. Sci. 110, E3265-E3270 (2013).

21. Ren, T. L., Tian, H., Xie, D. \& Yang, Y. Flexible Graphite-on-Paper Piezoresistive Sensors. Sensors 12, 6685-6694 (2012).

22. Wang, Y. \& Zhou, H. To draw an air electrode of a Li-air battery by pencil. Energy Environ. Sci. 4, 1704-1707 (2011).

23. Kurra, N., Dutta, D. \& Kulkarni, G. U. Field effect transistors and RC filters from pencil-trace on paper. Phys Chem Chem Phys 15, 8367-8372 (2013).

24. Kurra, N. \& Kulkarni, G. U. Pencil-on-paper: electronic devices. Lab Chip 13, 2866-2873 (2013). 
25. ul Hasan, K., Nur, O. \& Willander, M. Screen printed ZnO ultraviolet photoconductive sensor on pencil drawn circuitry over paper. Appl. Phys. Lett. 100, 211104 (2012).

26. Mandal, P., Dey, R. \& Chakraborty, S. Electrokinetics with "paper-and-pencil" devices. Lab Chip 12, 4026-4028 (2012).

27. Favier, F., Walter, E. C., Zach, M. P., Benter, T. \& Penner, R. M. Hydrogen Sensors and Switches from Electrodeposited Palladium Mesowire Arrays. Science 293, 2227-2231 (2001).

28. Lonergan, M. C. et al. Array-Based Vapor Sensing Using Chemically Sensitive, Carbon Black-Polymer Resistors. Chem. Mater. 8, 2298-2312 (1996).

29. Doleman, B. J., Lonergan, M. C., Severin, E. J., Vaid, T. P. \& Lewis, N. S. Quantitative Study of the Resolving Power of Arrays of Carbon Black-Polymer Composites in Various Vapor-Sensing Tasks. Anal. Chem. 70, 4177-4190 (1998).

30. Mark, J. E. Polymer data handbook. (Oxford University Press, 1999).

\section{Acknowledgments}

The authors would like to thank Jiayan Luo for his assistance in obtaining SEM micrographs; Alexander Smith for his help in acquisition and interpretation of EDS spectra; Ching-Hsuan Wu for the Stereomicroscope images; and Yi-Kai Huang and Dr. Kalyan Raidongia for valuable discussions. Financial support was partially provided by NSF through a CAREER award (DMR 0955612, REU supplement for Z.Z.), and the Sony Corporation through a gift donation. J.K. thanks the support from the Ryan Fellowship and the International Institute for Nanotechnology at Northwestern. J.H. was an Alfred P. Sloan Research Fellow and held a fellowship with Northwestern University's Searle Center for Advancing Learning and Teaching during the course of this work.

\section{Author contributions}

C.W.L. designed the strain gauge. C.W.L. and Z.Z. performed the experiments and analyzed the data. J.H. conceived the idea and oversaw the project with help of J.K. All authors wrote the paper, discussed the results, and commented on the manuscript.

\section{Additional information}

Competing financial interests: The authors declare no competing financial interests. How to cite this article: Lin, C.-W., Zhao, Z., Kim, J. \& Huang, J. Pencil Drawn Strain Gauges and Chemiresistors on Paper. Sci. Rep. 4, 3812; DOI:10.1038/srep03812 (2014) (c) (1) (2) (2) This work is licensed under a Creative Commons Attribution-
NonCommercial-ShareAlike 3.0 Unported license. To view a copy of this license,
visit http://creativecommons.org/licenses/by-nc-sa/3.0 